\title{
Condilomas Anogenitais numa Consulta de Doenças Sexualmente Transmissíveis: Centro de Saúde da Lapa - Lisboa, 2008 a 2014
}

\section{Anogenital Warts in a Major Venereology Clinic: Centro de Saúde da Lapa - Lisbon, 2008 to 2014}

\author{
Ana GAMEIRO $\triangle^{1}$, João ALVES², Irene SANTO³, Jacinta AZEVEDO ${ }^{3}$
}

Acta Med Port 2016 Feb;29(2):101-106 - http://dx.doi.org/10.20344/amp.6402

\section{RESUMO}

Introdução: O vírus do papiloma humano é responsável pela infeção sexualmente transmissível mais comum, podendo manifestar-se por um conjunto amplo de doenças, nomeadamente condilomas anogenitais, papilomatose laríngea recorrente, e neoplasias da região anogenital, colo do útero e orofaringe. Estima-se que os condilomas anogenitais afetem $1 \%$ da população sexualmente ativa, causados em cerca de $90 \%$ pelos genótipos 6 e 11 .

Material e Métodos: Identificámos os doentes com primeiro diagnóstico de condilomas anogenitais da consulta de doenças sexualmente transmissíveis do Centro de Saúde da Lapa, entre janeiro de 2008 e dezembro de 2014, e caracterizámos os doentes por sexo, orientação sexual, idade, localização das lesões, e número de parceiros nos últimos seis meses.

Resultados: Foram identificados 902 indivíduos com primeiro diagnóstico de condilomas anogenitais. Observámos uma diminuição significativa de novos casos nas mulheres com < 19 anos $(r=-0,848 ; p=0,016)$, e uma diminuição sem significado estatístico nos homens com $<19$ anos e nas mulheres entre os $20-24$ anos.

Discussão: Em outubro de 2008, a vacina quadrivalente (genótipos 6, 11, 16, 18) foi introduzida no plano nacional de vacinação de Portugal, abrangendo as adolescentes com 13 anos, com um catch-up para as de 17 anos. A diminuição de primeiros diagnósticos de condilomas anogenitais nas mulheres, deve-se provavelmente à sua vacinação antes de iniciarem a vida sexual.

Conclusão: Este estudo reforça a importância do programa nacional de vacinação para o vírus do papiloma humano.

Palavras-chave: Condiloma Acuminado; Doenças Virais Sexualmente Transmissíveis; Infecções por Papillomavirus; Vacina Quadrivalente Recombinante Contra Papilomavírus Humano, Tipos 6, 11, 16 e 18; Verrugas.

\section{ABSTRACT}

Introduction: Human papillomavirus infection is the most common sexual transmitted infection in the world, being associated with different diseases, namely anogenital warts, recurrent respiratory papillomatosis and anal, cervical, and oropharyngeal cancers. Among sexually active people, approximately $1 \%$ has anogenital warts, $90 \%$ of cases resulting from genotypes 6 and 11 .

Material and Methods: Patients diagnosed with first episode of anogenital warts from 2008 to 2014 in Lisbon's major venereology clinic were identified, and characterized according to sex, sexual orientation, age, warts location, and number of sexual partners.

Results: We observed a statistically significant decrease in first anogenital warts diagnosis among $<19$ year old females $(r=-0.848$; $p=0.016$ ) and a non-statistically significant decrease among < 19 - year-old males and among $20-24$ year old females.

Discussion: In October 2008, the quadrivalent vaccine (genotypes $6,11,16,18$ ) was introduced in the Portuguese national vaccination program, targeting 13-year-old females, with a catch-up for 17 year old females. In the women's group, decrease in first anogenital warts diagnosis is probably related to human papillomavirus vaccination before onset of sexual activity.

Conclusion: This study reinforces the importance of national human papillomavirus vaccination program.

Keywords: Condylomata Acuminata; Human Papillomavirus Recombinant Vaccine Quadrivalent, Types 6, 11, 16, 18; Papillomavirus Infections; Sexually Transmitted Diseases, Viral; Warts.

\section{INTRODUÇÃO}

O vírus do papiloma humano (HPV) é responsável pela infeção sexualmente transmissível (IST) mais comum, podendo manifestar-se por um conjunto amplo de doenças, nomeadamente condilomas anogenitais (CAG), papilomatose laríngea recorrente, e neoplasias da região anogenital, colo do útero e orofaringe. ${ }^{1}$

A grande família do HPV é constituída por mais de 120 genótipos, em que cerca de 40 apresentam tropismo para os genitais e região anal. ${ }^{2}$ Estima-se que os CAG afectem $1 \%$ da população sexualmente ativa, $90 \%$ resultantes da infeção pelos genótipos de baixo risco 6 e $11{ }^{3.6}$

A transmissão do HPV ocorre por contacto pele-pele, pele-mucosa e mucosa-mucosa. O coito não é requisito para a transmissão, pelo que a proteção conferida pelo preservativo é parcial. ${ }^{7}$ Factores de risco conhecidos da infeção por HPV são: início precoce da vida sexual, múltiplos parceiros, história de outras infeções sexualmente transmissíveis (IST), tabagismo e gravidez precoce. ${ }^{7}$

Em outubro de 2008, a vacina quadrivalente (genótipos $6,11,16,18)$ foi introduzida no plano nacional de vacinação de Portugal (PNV), com indicação para adolescentes com 13 anos. Associadamente, entre 2009 e 2011, decorreu uma campanha de vacinação para mulheres com 17 anos. ${ }^{8}$ Os objectivos do presente estudo são: avaliar a po-

1. Serviço de Dermatologia. Centro Hospitalar e Universitário de Coimbra. Coimbra. Portugal.

2. Serviço de Dermatologia. Hospital Garcia da Orta. Almada. Portugal.

3. Consulta de Doenças Sexualmente Transmissíveis. Centro de Saúde da Lapa. Lisboa. Portugal.

$\triangle$ Autor correspondente: Ana Gameiro. anaportelinhag@gmail.com

Recebido: 17 de março de 2015 - Aceite: 03 de agosto de 2015 | Copyright $\odot$ Ordem dos Médicos 2016 
pulação de doentes com primeiro diagnóstico de CAG na consulta de doenças sexualmente transmissíveis (DST) do Centro de Saúde da Lapa (CSL), o maior centro de IST em Portugal, entre 2008 e 2014, e caracterizar os doentes em relação ao sexo, idade, orientação sexual, localização das lesões, e número de parceiros.

\section{MATERIAL E MÉTODOS}

A consulta de DST do CSL caracteriza-se pela sua grande acessibilidade, é gratuita, e não requer agendamento prévio. Realizámos um estudo retrospectivo observacional, identificámos os doentes com primeiro diagnóstico de CAG entre janeiro de 2008 e dezembro de 2014, e caracterizámos os doentes por sexo, orientação sexual, idade, localização das lesões, e número de parceiros nos últimos seis meses.

O coeficiente de correlação de Pearson foi utilizado para avaliar o grau de correlação entre: percentagem de casos com primeiro diagnóstico de CAG na consulta e anos de estudo (2008 - 2014). A análise estatística foi realizada

Tabela 1 - Correlação de Pearson da percentagem de primeiro diagnóstico de CAG, 2008 - 2014

\begin{tabular}{lll}
\hline Mulheres com < 19 A & Correlação de Pearson & $-0,848$ \\
& $p$ & 0,016 \\
Homens com < 19 A & Correlação de Pearson & $-0,605$ \\
& $p$ & 0,150 \\
Mulheres 19 - 24 A & Correlação de Pearson & $-0,410$ \\
& $p$ & 0,361 \\
Homens 19-24 A & Correlação de Pearson & $-0,349$ \\
& $p$ & 0,443 \\
\hline
\end{tabular}

$25,00 \%$

$20,00 \%$

$\%$

$15,00 \%$

com o programa estatístico IBM SPSS, versão 22. Foi considerado como nível de significância estatística $p<0,05$.

\section{RESULTADOS}

Entre 2008 e 2014, foram realizados 902 primeiros diagnósticos de CAG, $560(62,1 \%)$ homens e 342 (37,9\%) mulheres. A percentagem de homens que fazem sexo com homens $(\mathrm{HSH})$ nos doentes com primeiro diagnóstico de CAG foi de $18,4 \%$.

O número absoluto de doentes com primeiro diagnóstico de CAG diminuiu de 2008 a 2014, mas o número de primeiras consultas por ano também diminuiu, tendo sido respectivamente: 1849 em 2008, 2047 em 2009, 1709 em 2010, 1652 em 2011, 1337 em 2012, 1545 em 2013, e 1315 em 2014.

Considerando a diminuição do número de consultas, verificou-se apenas um ligeiro decréscimo no número de primeiros diagnósticos de CAG por número de primeiras consultas (excluindo as demais consultas realizadas nesse ano, para um mesmo doente). Por conseguinte, a percentagem de doentes com primeiro diagnóstico de CAG na consulta foi de: $8,22 \%(n=152)$ em $2008,7,38 \%(n=151)$ em $2009,8,78 \%$ ( $n=150)$ em $2010,7,99 \%(n=132)$ em $2011,9,05 \%(n=121)$ em 2012, 6,86\% ( $n=106)$ em 2013 , e 6,84\% ( $n=90)$ em 2014 .

Em 2013, a diminuição de primeiros diagnósticos de CAG resultou da diminuição de casos no sexo feminino $(5,98 \%(n=29))$, ao invés de uma percentagem mantida para o sexo masculino $(7,26 \%(n=77))$. Em 2014, a percentagem de primeiros diagnósticos de CAG nas mulheres foi superior à dos homens $(7,75 \%(n=31)$ vs $6,45 \%(n=$ 51)). Considerando a população com primeiro diagnóstico de CAG, observamos uma maior percentagem de casos nos jovens, diminuindo ao longo da vida. No entanto, nos

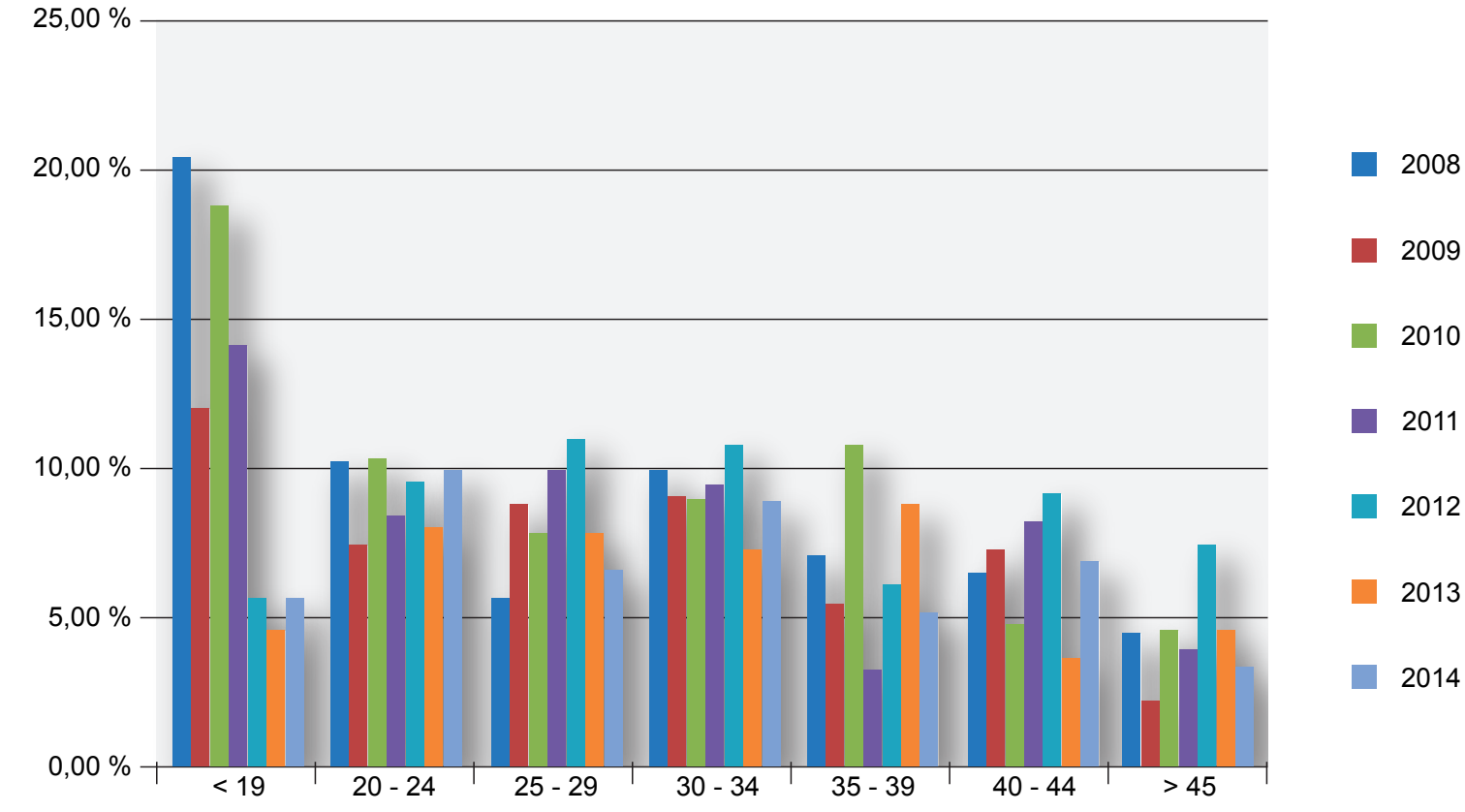

Figura 1 - Distribuição por classes etárias da incidência de $1^{\circ}$ diagnóstico de CAG, 2008 - 2014 


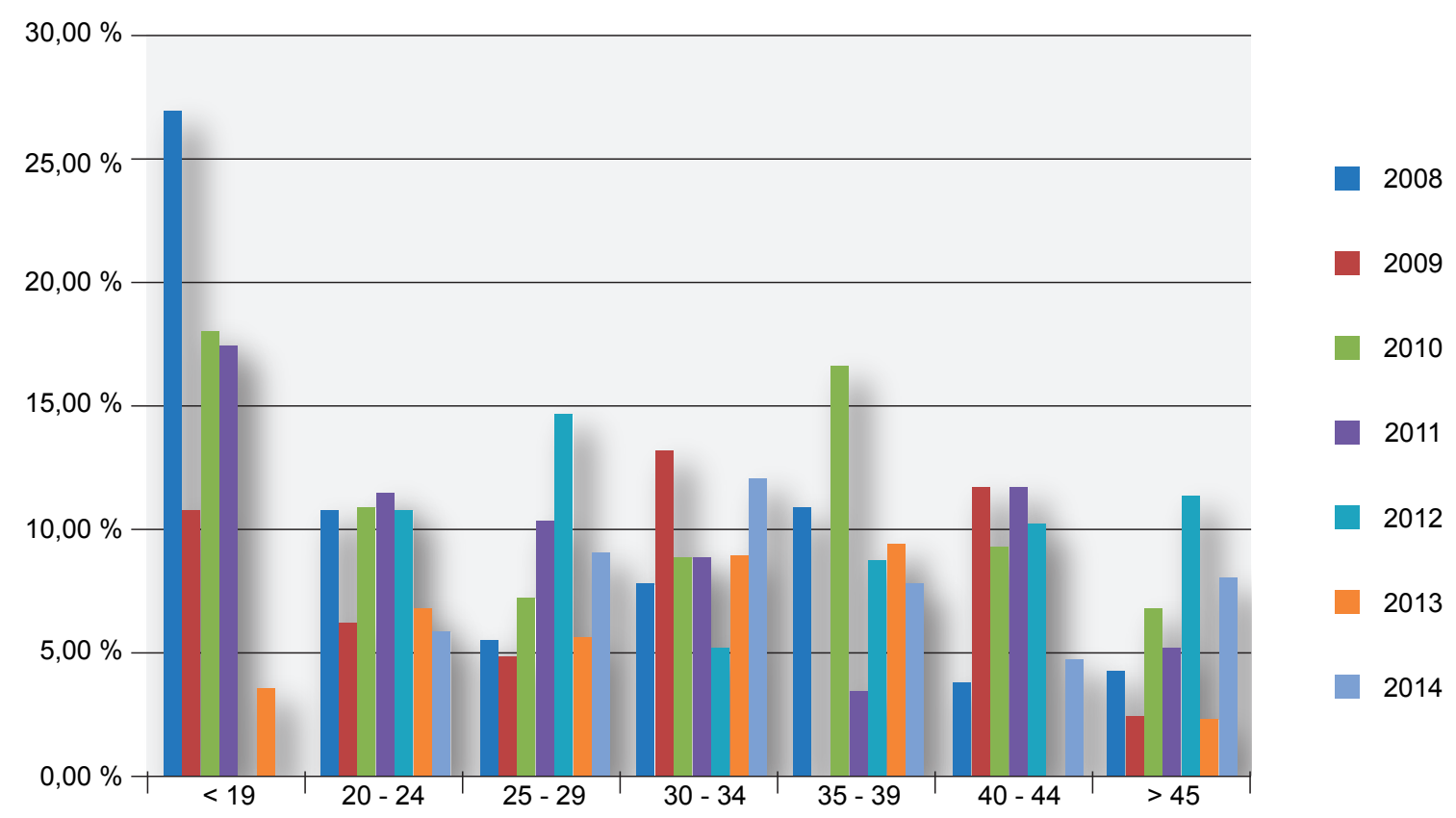

Figura 2 - Distribuição por classes etárias da incidência de 1º diagnóstico de CAG no sexo feminino, 2008 - 2014

últimos três anos (2012 - 2014) observa-se uma redução evidente do número de novos casos na classe etária $<19$, particularmente acentuada no sexo feminino (Fig.s 1, 2 e 3). Em 2012 e 2014, não houve registo de primeiro diagnóstico de CAG em mulheres com < 19 anos, e em 2013 a percentagem foi de $3,57 \%$ (representando duas mulheres de nacionalidade brasileira). No grupo de mulheres com < 19 anos, a percentagem de doentes com primeiro diagnóstico de CAG diminuiu significativamente $(r=-0,848 ; p=$ 0,016 ) ao longo dos anos do estudo, e no grupo de homens com < 19 anos, diminuiu também, mas sem significado estatístico (Tabela 1).

Observamos também que em 2013 e 2014, nas muIheres da classe etária 20 - 24 anos, a percentagem de doentes com primeiro diagnóstico de CAG diminuiu comparativamente aos anos anteriores, sendo respectivamente $6,85 \%$ e 5,94\% (Fig. 2). Nessa classe etária, seis das nove mulheres diagnosticadas em 2013, e três das seis mulheres diagnosticadas em 2014, não eram de nacionalidade portuguesa. De salientar que a representação na consulta de mulheres das classes etárias < 19 e entre 20 - 24 anos foi relativamente constante, de $28,87 \%$ (2012) a $41,1 \%$ (2009) de todas as mulheres observadas.

As localizações dos CAG foram registadas em 794 dos 902 doentes, subdividindo-se em lesões perianais, do pénis e da vulva. As lesões perianais foram identificadas em 141 casos, 19,1\% (27) mulheres e 80,1\% (114) homens, destes $78,1 \%$ (89) HSH. As lesões localizadas ao pénis foram identificadas em 369 doentes, destes 14,4\% (53) HSH. As lesões da vulva foram identificadas em 302 casos.

Em relação ao número de parceiros sexuais nos seis meses prévios ao diagnóstico de CAG, 35 dos doentes ne- garam parceiros, 511 referiram um parceiro, 264 referiram dois a cinco parceiros, e 63 referiram mais de cinco parceiros. Esse dado não foi registado em 29 doentes.

\section{DISCUSSÃO}

A percentagem de doentes com primeiro diagnóstico de CAG no CSL, entre 2008 e 2014 , foi relativamente constante, variando de $6,84 \%$ a $9,05 \%$.

Os diferentes estudos epidemiológicos publicados apresentam variações marcadas nas taxas de incidência/ prevalência de CAG, e de infeção por HPV com tropismo genital, que poderão ser reflexo dos comportamentos sexuais das diferentes populações. No entanto, estes estudos variam tanto na metodologia, como na apresentação dos dados, dificultando a apreciação do impacto global das IST por HPV. ${ }^{5,11-13}$ Como exemplo, em 1997, Munk et al reportaram uma prevalência de CAG de $17 \%$ nas mulheres entre os 17 - 24 anos na Dinamarca. ${ }^{9}$ Em 2007, um estudo multicêntrico, envolvendo países nórdicos (amostra de 70000 mulheres), mostrou uma prevalência de 10,6\% de CAG ao longo da vida. ${ }^{6}$ Em 2011, no Reino Unido, a pesquisa de DNA de HPV na urina foi positiva em $29 \%$ das muIheres e $18 \%$ dos homens. ${ }^{7}$ Outros países, nomeadamente a Eslovénia, reportaram incidências cumulativas de CAG muito inferiores, de cerca de $0,35 \%$. $^{10}$

A incidência de CAG é habitualmente superior nos jovens, com posterior diminuição ao longo da vida, justificada pelo maior número de parceiros sexuais nos jovens, e consequentes infeções por HPV. ${ }^{5} \mathrm{Na}$ população estudada, observamos também um maior número de primeiros diagnósticos de CAG nos jovens, diminuindo ao longo da vida em ambos os sexos. Todavia, nos últimos três anos, nas 


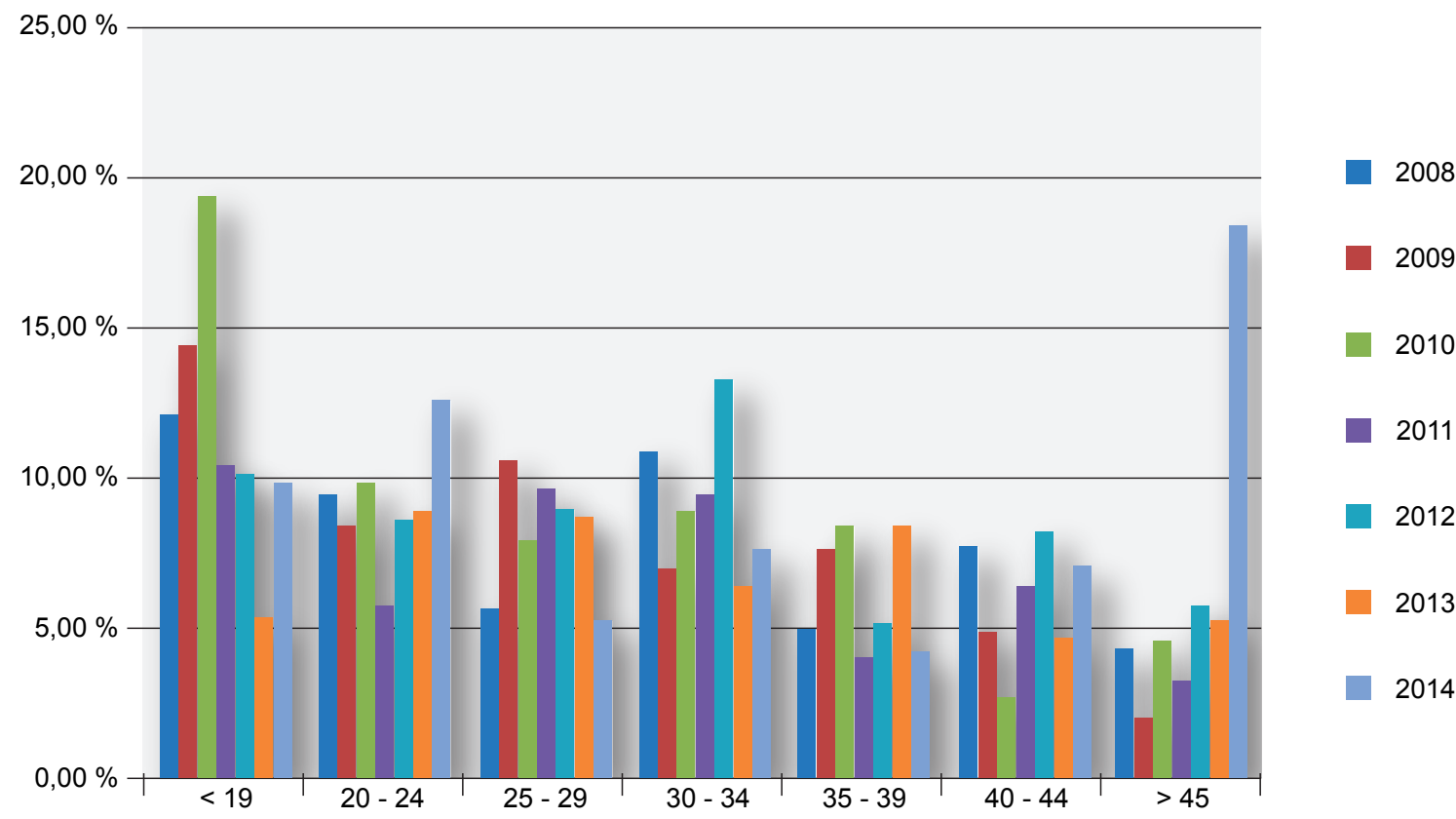

Figura 3 - Distribuição por classes etárias da percentagem de 1º diagnóstico de CAG no sexo masculino, 2008 - 2014

mulheres e homens com < 19 anos, e nos últimos dois anos, nas mulheres entre 20 - 24 anos, a percentagem de doentes com primeiro diagnóstico de CAG encontra-se notoriamente diminuída em relação a anos anteriores. Nas mulheres com < 19 e entre 20 - 24 anos, essa percentagem é inclusivamente menor que nas faixas etárias superiores, e a diminuição de primeiros diagnósticos de CAG no grupo de mulheres com < 19 anos mostrou-se ser estatisticamente significativa.

Dados da Direção Geral de Saúde mostram que as taxas de vacinação para o HPV das mulheres vacinadas aos 13 anos foram de aproximadamente $90 \%$, e para as mulheres abrangidas durante o catch-up de aproximadamente $85 \% .^{8}$ A diminuição de primeiros diagnósticos de CAG nas mulheres com < 19 anos e entre 20 - 24 anos é expectável, considerando que as primeiras mulheres vacinadas aos 13 anos, abrangidas pelo PNV têm atualmente 19 anos, e que as mulheres vacinadas durante o catch-up têm atualmente idade máxima de 23 anos.

Nas mulheres da classe etária 20 - 24, a diminuição não se mostrou estatisticamente significativa, o que pode ser justificado pela menor taxa de vacinação em esquema de catch-up; por parte das mulheres dessa classe etária não terem sido vacinadas no âmbito do PNV; e pela elevada proporção de doentes de outras nacionalidades (41,3\%), em a vacina para o HPV não era incluída nos programas nacionais de vacinação, como Brasil e PALOP.

Não foi registado de forma sistemática o status vacinal dos doentes com primeiro diagnóstico de CAG, mas avaliámos caso a caso o status vacinal das mulheres com primeiro diagnóstico de CAG e < 19 e 20 - 24 anos, nos últimos dois anos (2013 - 2014). Todas as mulheres de nacionalidade portuguesa $(n=6)$ recusaram a vacinação para o HPV, à exceção de uma mulher, da qual se desconhece o status vacinal. No caso das mulheres de nacionalidade estrangeira $(n=11)$, na sua maioria não foi possível confirmar o seu status vacinal, no entanto, as duas mulheres diagnosticadas com < 19 anos eram de nacionalidade brasileira, e não tinham sido vacinadas.

Uma das limitações do presente estudo é incluir a população de um centro urbano, uma vez que a prevalência de CAG, bem como de outras IST, é mais elevada nos centros urbanos que nas áreas rurais. Outras limitações prendem-se por ser relativo a uma consulta específica de DST, apresentando uma maior incidência de IST que a população geral, e por ser um estudo retrospectivo. Outro factor que reconhecemos importante enumerar trata-se da modificação do regime de acesso à consulta durante quatro meses em 2012, altura em que foram instituídas taxas moderadoras. Nesse ano registou-se uma diminuição do número total de primeiras consultas, contudo, o número de novas consultas em 2012 ( $n=1337$ ) foi equiparável a 2014 ( $n=1315)$.

As terapêuticas para os CAG subdividem-se em: terapêuticas executadas exclusivamente pelo médico, como a crioterapia, electrocirurgia e o ácido tricloroacético, e em terapêuticas tópicas de aplicação pelo próprio doente, estando em Portugal apenas disponível o imiquimod. Os diferentes tratamentos mostram-se comparáveis em termos de eficácia e taxas de recorrência, mas as taxas de recorrência são elevadas, estimadas em 30\% - 70\% aos seis meses, justificado pelos tratamentos serem focados na destruição local das lesões, não se eliminando a infeção pelo vírus. ${ }^{5}$ Assim, apesar da progressão indolente, os tratamentos prolongados e as elevadas taxas de recidiva condicionam um impacto socioeconómico elevado. ${ }^{1}$

A abordagem mais eficaz é profilática, a redução do 
número de parceiros, a utilização de preservativo e, atualmente, a vacinação para o HPV antes de iniciar a atividade sexual. A vacina Gardasil $^{\circledR}$, é uma vacina quadrivalente para os genótipos $6,11,16,18$ do HPV. A vacina consiste na proteína L1 do vírus (proteína principal), agregada a outras partículas virais recombinantes não infeciosas, e induz a formação de anticorpos em títulos cerca de 60 - 100 vezes superiores que na infeção natural, com manutenção de títulos elevados durante pelo menos cinco anos. ${ }^{14,15}$

A Austrália foi o primeiro país a incluir a vacinação para o HPV no seu programa de vacinação em abril de 2007, e a reportar a diminuição da incidência de CAG após a introdução da vacina para o HPV. ${ }^{16-18}$ Posteriormente, outros países reportaram a diminuição da incidência de CAG, como a Dinamarca ${ }^{19}$ a Suécia ${ }^{20}$ e os Estados Unidos. ${ }^{21,22}$ Em outros países, como na Alemanha, em que as taxas de vacinação são baixas ( $\approx 40 \%$ ), não se observa diminuição na incidência de CAG. ${ }^{23}$ Apresentamos o primeiro estudo em Portugal que evidencia o impacto positivo da vacinação na diminuição de CAG. ${ }^{24}$

Considera-se que o tempo de latência entre a infeção por HPV e as lesões de CAG é em média 3 - 8 meses, pelo que a história natural dos CAG permite comprovar a curto prazo a eficácia conferida pela vacina. ${ }^{5}$ No entanto, a eficácia em relação ao cancro colo do útero poderá apenas ser observada a longo prazo, uma vez que o tempo de evolução para esta neoplasia é cerca de 20 anos. Apesar disso, nos Estados Unidos, observou-se já uma diminuição no número de neoplasias intraepiteliais do colo do útero e do canal anal nas mulheres vacinadas. ${ }^{25}$

Na Austrália, a concomitante diminuição da incidência de CAG nos homens heterossexuais não vacinados para o HPV foi interpretada como 'efeito de grupo' por interposta vacinação das mulheres. ${ }^{16-18} \mathrm{O}$ presente estudo observa também uma diminuição de CAG nos homens com < 19 anos, contudo sem significado estatístico. São necessários estudos mais alargados que clarifiquem a diminuição de CAG nos homens em Portugal, que poderá ser também justificada por um 'efeito de grupo'.

Está estimado que aproximadamente $30 \%$ de todas a neoplasias relacionadas com o HPV ocorram em homens, e que a vacinação adicional do sexo masculino tem impacto na redução de neoplasias relacionadas com o HPV em ambos os sexos. ${ }^{26}$ Reconhece-se porém, que o benefício da vacinação do sexo masculino é mais acentuado em países onde os programas de vacinação das mulheres têm uma cobertura baixa da população. ${ }^{27}$ Como exemplo, na Nova Zelândia, a vacinação dos homens demonstrou não ser positiva em termos de custo-efetividade. ${ }^{28}$ No entanto, a população HSH não beneficia da imunidade de grupo e existe atualmente um debate ético em relação à extensão da vacinação ao sexo masculino. ${ }^{29-31}$

O Advisory Committee of Immunization Practices
(ACIP) recomenda a vacinação para o HPV nas mulheres e homens, entre os 11 e 12 anos. ${ }^{32}$ A recomendação para a vacinação dos homens tem sido adotada por vários países. ${ }^{14} \mathrm{Na}$ Austrália, a vacinação foi estendida aos rapazes entre os 12 - 13 anos, com catch-up para os com 14 - 15 anos em 2013. ${ }^{18}$

Atualmente a vacina nonavalente (HPV 6, 11, 16, 18, $31,33,45,52,58$ ) encontra-se aprovada pela Agência Europeia de Medicamentos. Esta visa a proteção para genótipos responsáveis por $90 \%$ dos casos de cancro do colo do útero (a vacina quadrivalente confere uma proteção de cerca de $70 \%$ ). Num futuro próximo, a vacina quadrivalente será provavelmente substituída pela vacina nonavalente..$^{14,34}$

\section{CONCLUSÃO}

Observámos uma diminuição significativa de primeiros diagnósticos de CAG em mulheres com < 19 anos na consulta de DST no Centro de Saúde da Lapa, provavelmente relacionada com o início da vida sexual após a vacinação para o HPV. Estes dados reforçam a importância da vacinação para o HPV.

São necessários dados epidemiológicos mais extensos para poder avaliar a eficácia da vacina para o HPV noutras patologias como o cancro do colo do útero, e no impacto por 'efeito de grupo' nos homens. Consideramos necessário repensar a população abrangida para a vacinação contra o HPV, e avaliar o custo-beneficio da vacinação nos homens em Portugal.

\section{AGRADECIMENTOS}

Os autores gostariam de agradecer a Margarida Marques, pela colaboração na análise estatística.

\section{PROTECÇÃO DE PESSOAS E ANIMAIS}

Os autores declaram que os procedimentos seguidos estavam de acordo com os regulamentos estabelecidos pelos responsáveis da Comissão de Investigação Clínica e Ética e de acordo com a Declaração de Helsínquia da Associação Médica Mundial.

\section{CONFIDENCIALIDADE DOS DADOS}

Os autores declaram ter seguido os protocolos do seu centro de trabalho acerca da publicação dos dados de doentes.

\section{CONFLITO DE INTERESSES}

Os autores declaram não ter nenhum conflito de interesses relativamente ao presente artigo.

\section{FONTES DE FINANCIAMENTO}

Não existiram fontes externas de financiamento para a realização deste artigo. 


\section{REFERÊNCIAS}

1. Tommasino M. The human papillomavirus family and its role in carcinogenesis. Semin Cancer Biol. 2014;26:13-21.

2. Kirnbauer R, Lenz P. Human papillomaviruses. In: Bolognia dermatology. $3^{\text {rd }}$ ed. Amsterdam: Elsevier Limited; 2012. p. 1303-19.

3. Moscicki AB, Schiffman M, Burchell A, Albero G, Giuliano AR, Goodman MT, et al. Updating the natural history of human papillomavirus and anogenital cancers. Vaccine. 2012;30:F24-33.

4. Chesson HW, Dunne EF, Hariri S, Markowitz LE. The estimated lifetime probability of acquiring human papillomavirus in the United States. Sex Transm Dis. 2014;41:660-4

5. Patel H, Wagner M, Singhal $P$, Patel S. Systematic review of the incidence and prevalence of genital warts. BMC Infect Dis. 2013;13:39.

6. Kjær SK, Tran TN, Sparen P, Tryggvadottir L, Munk C, Dasbach E, et al. The burden of genital warts: a study of nearly 70,000 women from the general female population in the 4 nordic countries. J Infect Dis. 2007; 196:1447-54

7. Johnson A, Mercer C, Beddows S, Silva N, Desai S, Howell-Jones R, et al. Epidemiology of, and behavioural risk factors for, sexually transmitted human papillomavirus infection in men and women in Britain. Sex Transm Infect. 2012;88:212-7.

8. Direção Geral de Saúde. A vacinação contra o vírus do papiloma humano (HPV) em Portugal. [consultado 2015 mar 16]. Disponíve em: http://www.dgs.pt/documentos-e-publicacoes/boletim-vacinacaoedicao-especial-abril-2014.aspx.

9. Munk C, Svare EI, Poll P, Bock JE, Kjaer SK. History of genital warts in 10,838 women 20 to 29 years of age from the general population. Risk factors and association with Papanicolaou smear history. Sex Transm Dis. 1997;24:567-72.

10. Klavs I, Grgic-Vitek M. The burden of genital warts in Slovenia: results from a national probability sample survey. Euro Surveill. 2008;6;13:pii:19032.

11. Castellsague $X$, Cohet $C$, Puig-Tintore L, Acebes L, Salinas J, Martin M, et al. Epidemiology and cost of treatment of genital warts in Spain. Eur J Public Health. 2009;19:106-10

12. Aubin $F$, Prétet $J$, Jacquard $A$, Saunier $M$, Carcopino $X$, Jaroud $F$, et al. Human papillomavirus genotype distribution in external acuminata condylomata: a large French national study. Clin Infect Dis. 2008; $1 ; 47: 610-5$

13. Borges da Costa J, Azevedo J, Santo I. Sexually transmitted infections and related sociodemographic factors in Lisbon's major venereology clinic: a descriptive study of the first 4 months of 2007. J Eur Acad Dermatol Venereol. 2010;24:811-4.

14. Chatterjee $A$. The next generation of HPV vaccines: nonavalent vaccine V503 on the horizon. Expert Rev Vaccines. 2014:13:1279-90.

15. Isidean SD, Tota JE, Gagnon JA, Franco EL. Human papillomavirus vaccines: key factors in planning cost-effective vaccination programs. Expert Rev Vaccines. 2014;30:1-15

16. Fairley CK, Hocking JS, Gurrin LC, Chen MY, Donovan B, Bradshaw CS Rapid decline in presentations of genital warts after the implementation of a national quadrivalent human papillomavirus vaccination programme for young women. Sex Transm Infect. 2009;85:499-502.

17. Ali H, Donovan B, Wand H, Read T, Regan D, Grulich A, et al. Genital warts in young Australian five years into national human papillomavirus vaccination programme: national surveillance data. BMJ 2013;346:f2032.

18. Harrison C, Britt H, Garland S, Conway L, Stein A, Pirotta M, et al Decreased management of genital warts in young women in australian general practice post introduction of national HPV vaccination program: results from a nationally representative cross-sectional general practice study. PLoS One. 2014;9:e105967.

19. Baandrup L, Blomberg M, Dehlendorff C, Sand C, Andersen K, Kjaer S. Significant decrease in the incidence of genital warts in young Danish women after implementation of a national human papillomavirus vaccination program. Sex Transm Dis. 2013:40:130-5.

20. Leval A, Herweijer E, Ploner A, Eloranta S, Fridman J, Dillner J, et al. Quadrivalent human papillomavirus vaccine effectiveness: a Swedish national cohort study. J Natl Cancer Inst. 2013;105:469-74.

21. Hariri S, Markowitz LE, Dunne EF, Unger ER. Population impact of HPV vaccines: summary of early evidence. J Adolesc Health. 2013;53:67982.

22. Hariri S, Bennett NM, Niccolai LM, Schafer S, Park IU, Bloch KC, et al. Reduction in HPV 16/18-associated high grade cervical lesions following HPV vaccine introduction in the United States - 2008-2012. Vaccine. 2015;33:1608-13.

23. Poethko-Müller C, Buttmann-Schweiger N; KiGGS Study Group. HPV vaccination coverage in German girls: results of the KiGGS study: first follow-up (KiGGS Wave1) Bundesgesundheitsblatt Gesundheitsforschung Gesundheitsschutz. 2014;57:869-77.

24. Pista A, Oliveira C, Cunha M, Paixão M, Real O. Prevalence of human papillomavirus infection in women in Portugal - The Cleopatre Portugal Study. Int J Gynecol Cancer. 2011;21:1150-8.

25. Hariri S, Bennett NM, Niccolai LM, Schafer S, Park IU, Bloch KC, et al. Reduction in HPV 16/18-associated high grade cervical lesions following HPV vaccine introduction in the United States - 2008-2012. Vaccine, 2015;33:1608-13.

26. Marty R, Roze S, Bresse X, Largeron N, Smith-Palmer J. Estimating the clinical benefits of vaccinating boys and girls against HPV-related diseases in Europe. BMC Cancer. 2013;13:10.

27. D'Souza G, Dempsey A. The role of HPV in head and neck cancer and review of the HPV vacine. Prev Med. 2011;53:S5-11.

28. Pearson AL, Kvizhinadze G, Wilson N, Smith M, Canfell K, Blakely T. Is expanding HPV vaccination programs to include school-aged boys likely to be value-for-money: a cost-utility analysis in a country with an existing school-girl program. BMC Infect Dis. 2014;14:351

29. Wang Z, Mo PK, Lau JT, Lau M, Lai CH. Acceptability of HPV vaccines and perceptions related to genital warts and penile/anal cancers among men who have sex with men in Hong Kong. Vaccine. 2013;31:4675-81.

30. Brown B, Davtyan M, Leon SR, Sanchez H, Calvo G, Klausner JD, et al. A prospective cohort study characterising the role of anogenital warts in HIV acquisition among men who have sex with men: a study protocol. BMJ Open. 2014;4:e005687.

31. Giacomet V, Penagini F, Trabattoni D, Viganò A, Rainone V, Bernazzan $\mathrm{G}$, et al. Vaccine. Safety and immunogenicity of a quadrivalent human papillomavirus vaccine in HIV-infected and HIV-negative adolescents and young adults. Vaccine. 2014;32:5657-61.

32. Centers for Disease Control and Prevention. Human papillomavirus vaccination: recommendations of the Advisory Committee on Immunization Practices (ACIP). August 29, 2014 / 63 (RR05); 1-30. [consultado 2015 mar 16]. Disponível em: http://www.cdc.gov/mmwr/ preview/mmwrhtml/rr6305a1.htm

33. Stanley M. HPV vaccination in boys and men. Hum Vaccin Immunother. 2014;10:2109-11.

34. Petrosky E, Bocchini JA Jr, Hariri S, Chesson H, Curtis CR, Saraiya $M$, et al. Use of 9-valent human papillomavirus (HPV) vaccine: updated HPV vaccination recommendations of the advisory committee on immunization practices. MMWR Morb Mortal Wkly Rep. 2015;64:300-4. 